\title{
Methylation of 2-Naphthol Using Dimethyl Carbonate under Continuous-Flow Gas-Phase Conditions
}

\author{
Pietro Tundo, * Anthony E. Rosamilia, and Fabio Aricò \\ La Chimica per L'Ambiente, Consorzio Interuniversitario Nazionale, Via delle Industrie 21/8, \\ Marghera (VE), 30175, Italy \\ *tundop@unive.it
}

Green chemistry is an area of chemistry that is used in, but not exclusive to, the field of synthetic chemistry. In synthetic chemistry, green chemistry aims to use processes that reduce hazards to humankind and the environment and also reduce the quantity of waste produced. The 12 principles of green chemistry outlined by Anastas and Warner detail the general approach one should adopt for any reaction or synthetic strategy $(1-3)$. The study of cleaner chemistry methodologies in academia is integral for the development of green chemistry (4-8). However, for green chemistry to be successful, its ideals must be adopted and employed in industry, where chemical processes occur on a large scale (9).

In the chemical industry, reactions are generally performed using either batch or continuous-flow methods. Batch reactions in industry are analogous to laboratory reactions that use an autoclave or round-bottom flask, albeit more complicated. The relatively simple setup allows for many different types of reactions to be carried out. However, the limiting factor of batch reactions, as implied by the name, is that reactions are performed in batches and reaction volumes are limited to the size of the reactor. Conversely, continuous-flow systems are capable of incorporating larger reaction volumes. Substrates and auxiliary components are passed through the reaction site and expelled after the chemical transformation. The process occurs in a continuous manner and can provide access to large quantities of product in which operating efficiency is maximized (10). Most reactions in industry are performed using continuous-flow methods (11).

Despite the use of continuous-flow systems in industry, the analogous setup in research or teaching laboratories is not commonly found. Demonstration of this technique offers valuable hands-on experience while affirming the theoretical concepts that are valuable to industry. Adding to the educational chemistry literature, which offers various examples of continuous-flow systems $(12-18)$, the following experiment describes an example of a continuous-flow gas-phase reaction at atmospheric pressure. Dimethyl carbonate (DMC) is used as an alternative green methylating agent. The involvement of green chemistry and advanced concepts in this experiment make it suitable for organic chemistry students in the final year of their undergraduate studies.

\section{Dimethyl Carbonate as a Green Methylating Agent}

Dimethyl carbonate, an ambident electrophile, has recently been used a methylating and carboxymethylating reagent. Different reaction conditions can be used, that is, altering reaction temperature, to affect the reaction outcome $(19,20)$. In the case of methylation reactions, DMC is considered a greener alternative to other methylating agents such as methyl halides and dimethyl sulfate $(21-24)$. In comparison, DMC has a negligible toxicity, reactions can be catalytic, and less co-product is produced (25). In addition, DMC is produced readily in bulk from renewable resources (26-28) and it is biodegradable.

\section{Experimental Procedure}

The laboratory experiment was divided into three parts: the preparation of the solid-supported catalyst, assembly of the continuous-flow gas-phase reactor, and the methylation of 2-naphthol with dimethyl carbonate using the prepared catalyst.

The preparation of the solid-supported catalyst was a relatively simple process. The $\mathrm{K}_{2} \mathrm{CO}_{3}(0.5 \mathrm{~g})$ and poly(ethylene)glycol $(2.5 \mathrm{~g})$ were dissolved in water and the solution was added to the solid support, $\alpha-\mathrm{Al}_{2} \mathrm{O}_{3}$ beads ( $47 \mathrm{~g}$ ). After mixing the components, water was removed by rotary evaporation and the residual solid was dried in a vacuum oven. Hands-on time for the preparation of the catalyst took less than an hour, but the catalyst should be dried overnight. Once prepared, the students packed the solidsupported catalyst $\left(1 \% \mathrm{~K}_{2} \mathrm{CO}_{3}\right)$ into a glass column, which constitutes the reaction chamber of the continuous-flow gas-phase reactor.

The reaction setup (Figure 1) included a liquid pump, which carried the reaction mixture into the reaction column containing the solid-supported catalyst that was heated to $180{ }^{\circ} \mathrm{C}$. The reaction column was heated by a heating arm that wrapped around the glass column. Setup time for the equipment was about $1 \mathrm{~h}$. The temperature in the reaction column ensured that all substrates existed in the vapor phase. Under these conditions 2-naphthol ( $50 \mathrm{~g})$ was methylated by dimethyl carbonate $(625 \mathrm{~g})$. The gaseous substances were cooled and collected with a water condenser.

It is possible that some of the components of the reaction setup are not commonly found in undergraduate labs, such as a jacketed heating arm attached to a thermocouple (see the supporting information for more details) or a liquid pump; however, they should be readily attainable. With all the equipment, the reaction setup is easily manageable.

\section{Hazards}

The reaction is carried out at $180^{\circ} \mathrm{C}$, which presents a burn risk along with the possibility of solvent ignition (flash point of DMC is $18^{\circ} \mathrm{C}$ ) and increased pressure. Thus, the reaction setup should not be a closed system. DMC is a flammable liquid; therefore, experiments should be carried out in a fume hood and 


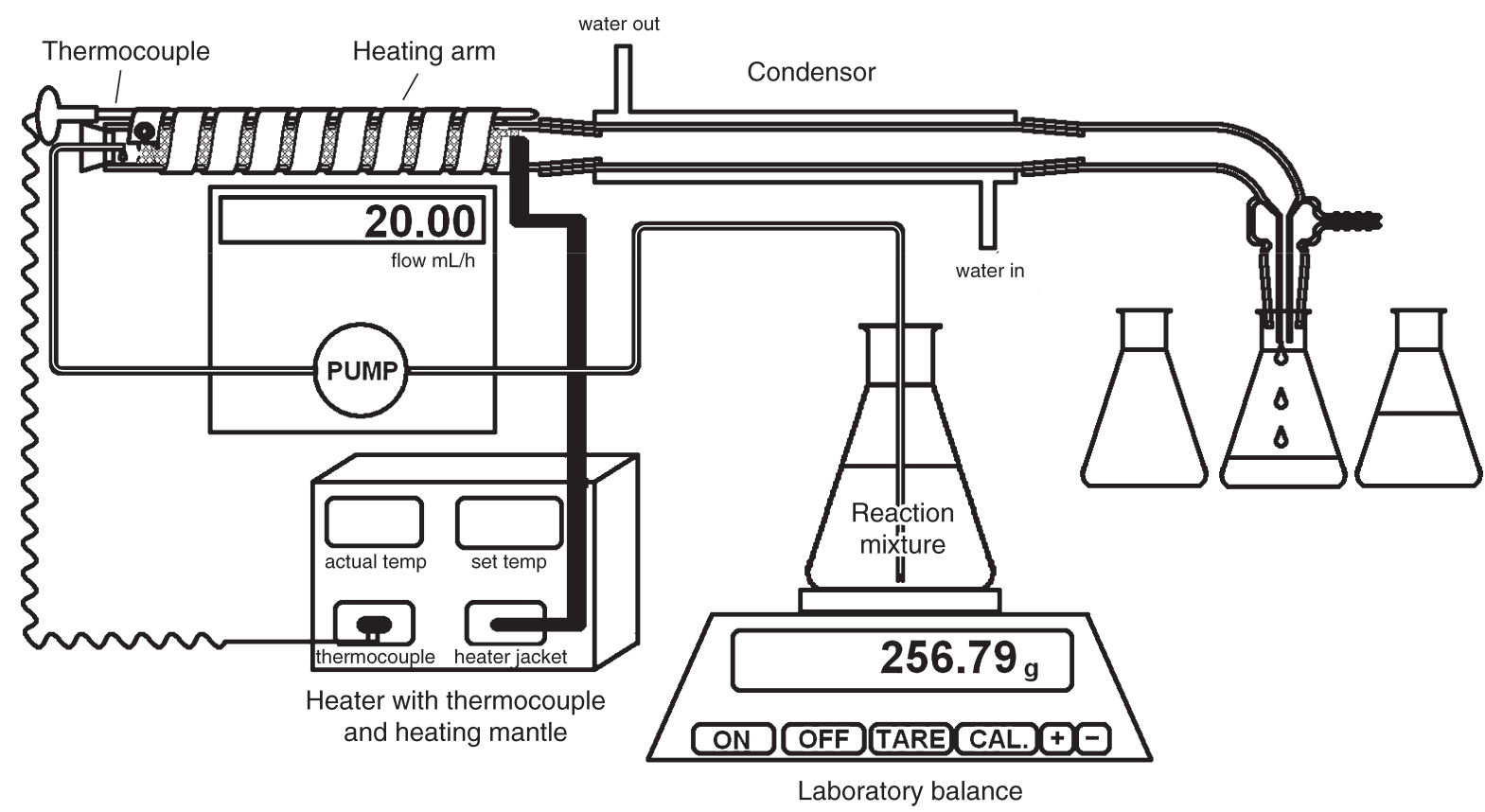

Figure 1. Setup of the continuous-flow reactor.

Scheme 1. Methylation of 2-Naphthol and the Base Catalytic Cycle

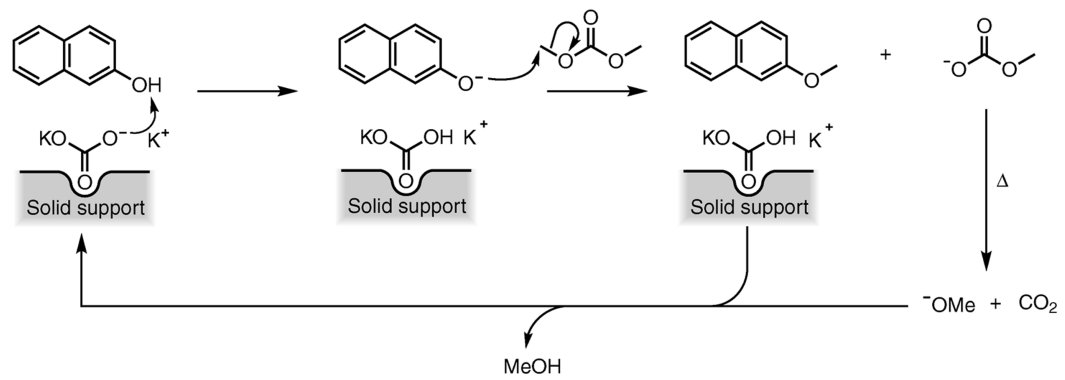

there should be no open flames or spark sources in the lab. Potassium carbonate is slightly corrosive and 2-naphthol is a mild irritant and is slightly corrosive. Methanol is extremely flammable and toxic by inhalation. Methanol may be fatal or cause blindness if swallowed. 2-Methoxynaphthalene is combustible and may act as an irritant.

\section{Results}

Using this continuous-flow reaction, the students achieved good conversions after $30 \mathrm{~min}$. A minimum conversion of $97 \%$ (by gas chromatography) into 2-methoxynaphthalene was obtained, although it was possible to leave the reaction for longer periods (up to days). The students evaporated the reaction solvent and analyzed the residue product via thin-layer chromatography, melting point, gas chromatography, and NMR spectrometry. The formation of 2-methoxynaphthalene was evidenced by the presence of a sweet-smelling aroma.

This experiment was conducted by a class of 20 students in their last year of undergraduate studies at a scientific high school. The student background included two yearlong courses in chemistry. Thus, they had a good notion of general chemistry, organic chemistry, and a base knowledge of laboratory instrumentation.
The class was divided into five groups of four students and each group was able to set up and correctly conduct the three parts of the experiment. During the experiment, the students were introduced to the concepts of continuous-flow synthesis and green chemistry. At the end of the experiment, they were questioned about the acquired notions and were able to answer readily and in a satisfactory way. This demonstrated that the students easily understand the concepts behind this experimental trial.

\section{Discussion}

Combining the two concepts, continuous-flow gas-phase chemistry and green chemistry, the students are exposed to principles that are useful in industry. In this experiment, DMC is used to methylate 2-naphthol under basic conditions (Scheme 1).

At $180{ }^{\circ} \mathrm{C}$, both 2 -naphthol and dimethyl carbonate are volatile and are present in the gas phase. The solid-supported potassium carbonate acts as the catalyst. The basic carbonate activates 2-naphthol via deprotonation, which produces the phenolate, a soft nucleophile. The phenolate reacts with the soft electrophilic center of DMC, the methyl group, to effect the methylation. The methylcarbonate anion produced decomposes to $\mathrm{CO}_{2}$ and methoxide. As the methoxide is a strong base, $\mathrm{CO}_{3}{ }^{2-}$ 
and methanol are regenerated from the deprotonation of $\mathrm{HCO}_{3}{ }^{-}$that was produced in the initial deprotonation. The products of the reaction are 2-methoxynaphthalene, methanol, and carbon dioxide, all volatile species that exit the continuousflow system. The lack of salt formation is pivotal to the success of the continuous-flow system. The analogous reaction with dimethyl sulfate would produce methylsulfonic acid as a co-product, which would deactivate the base, causing salt formation.

At the end of the reaction the methylated naphthol can easily be separated from the excess DMC and methanol (evaporation). The product, 2-methoxynaphthalene is an important fragrance (orange scent) and pharmaceutical intermediate.

\section{Conclusion}

The methylation of 2-naphthol with dimethyl carbonate was successfully performed using a continuous-flow gas-phase reaction. The reaction is green in terms of high yield, reduction in toxicity of the methylating agent, and reduction of waste. The laboratory exercise was successful in conveying the concept of continuous-flow systems to the student audience.

\section{Acknowledgment}

We would like to thank contributions from the Consorzio Interuniversitario Nazionale, La Chimica per l'Ambiente, INCA (Interuniversity National Consortium "Chemistry for the Environment").

\section{Literature Cited}

1. Anastas, P. T.; Warner, J. C. In Green Chemistry: Theory and Practice; Oxford University Press: New York, 1998.

2. Tundo, P.; Anastas, P.; Black, D. StC.; Breen, J.; Collins, T.; Memoli, S.; Miyamoto, J.; Polyakoff, M.; Tumas, W. Pure Appl. Chem. 2000, 72, 1207.

3. Tundo, P.; Perosa, A.; Zecchini, F. In Methods and Reagents for Green Chemistry: An Introduction; Wiley: New York, 2007.

4. Braun, B.; Charney, R.; Clarens, A.; Farrugia, J.; Kitchens, C.; Lisowski, C.; Naistat, D.; O'Neil, A. J. Chem. Educ. 2006, 83, 1126-1129.

5. Collins, T. J. J. Chem. Educ. 1995, 72, 965-966.

6. Reed, S. M.; Hutchison, J. E. J. Chem. Educ. 2000, 77, 1627-1629.

7. Sobral, A. J. F. N. J. Chem. Educ. 2006, 83, 1665-1666.
8. Mak, K. K. W.; Siu, J.; Lai, Y. M.; Chan, P.-K.J. Chem. Educ. 2006, 83, 943-946.

9. For example, industry in the United States alone released 7.1 billion pounds of more than 650 toxic chemicals into the environment in 2000. Doble, M.; Kruthiventi, A. K. Green Chemistry and Engineering. Elsevier: New York, 2007; pp 1-2.

10. Tundo, P. In Continuous Flow Methods in Organic Synthesis; Ellis Horwood Limited: Chichester, U.K., 1991.

11. Schmidt, L. D. In The Engineering of Chemical Reactions; Gubbins, K. E., Ed.; Oxford University Press: New York, 2005; p 87.

12. Bisson, P. J.; Whitten, J. E. J. Chem. Educ. 2006, 83, 1860-1863.

13. Iskander, M. N.; Jones, P. A. J. Chem. Educ. 1990, 67, 170-172.

14. Merino, J. M. J. Chem. Educ. 1992, 69, 754-756.

15. Englund, S. M. J. Chem. Educ. 1982, 59, 766-768.

16. Mattson, B.; Fujita, J.; Catahan, R.; Cheng, W.; Greimann, J.; Hoette, T.; Khandhar, P.; Mattson, A.; Rajani, A.; Sullivan, P.; Perkins, R. J. Chem. Educ. 2003, 80, 768-773.

17. Raymundo-Piñero, E.; Cazorla-Amorós, D. J. Chem. Educ. 1999, 76, 958-961.

18. Mattson, B.; Hulce, M.; Cheng, W.; Greimann, J.; Hoette, T.; Menzel, P. J. Chem. Educ. 2006, 83, 421-424.

19. Selva, M.; Tundo, P.; Perosa, A. J. Org. Chem. 2001, 66, 677-680.

20. Tundo, P.; Bressanello, S.; Loris, A.; Sathicq, G. Pure Appl. Chem. 2005, 77, 1719-1725.

21. March, J. In March's Advanced Organic Chemistry, Reaction, Mechanism, and Structure, 5th ed.; John Wiley \& Sons: New York, 2001; p 477.

22. Williamson, A. Justus Liebigs Ann. Chem. 1851, 77, 37.

23. Johnstone, R. A. W.; Rose, M. E. Tetrahedron 1979, 35, 2169.

24. Lewis, H. F.; Shaffer, S.; Trieschmann, W.; Cogan, H. Ind. Eng. Chem. 1930, 22, 34.

25. Tundo, P.; Selva, M. Acc. Chem. Res. 2002, 35, 706.

26. Romano, U.; Rivetti, F.; Di Muzio, N. U.S. Patent 4,318,862, 1981, C.A. 80141 (1979).

27. Delledonne, D.; Rivetti, F.; Romano., U. J. Organomet. Chem. 1995, C15, 448.

28. Nisihra, K.; Mizutare, K.; Tanaka, S. Process for Preparing Diester of Carbonic Acid. EP Patent Appl. 425197.

\section{Supporting Information Available}

Student handout; instructor notes; NMR spectra. This material is available via the Internet at http://pubs.acs.org. 